\title{
VOF-based simulation of conjugate mass transfer from freely moving fluid particles
}

\author{
A. Alke ${ }^{1}$, D. Bothe ${ }^{1}$, M. Kroeger ${ }^{1}$ \& H.-J.Warnecke ${ }^{2}$ \\ ${ }^{1}$ Center of Smart Interfaces, Technical University Darmstadt, Germany \\ ${ }^{2}$ Department of Chemical Engineering, University Paderborn, Germany
}

\begin{abstract}
In this paper two variants of a VOF-based approach for the numerical simulation of the molar mass transport of a diluted species in two-phase flows with deformable interfaces are introduced and compared. The variants differ in the manner of the computation of the mass transfer flux across the interface. The method assumes local thermodynamical equilibrium at the interface and enables the simulation of conjugated mass transfer problems across deformable interfaces, where the mass transport resistance lies in both phases. The considered model also allows for arbitrary distribution coefficients. First numerical simulations show the potential and the present limits of this method.

Keywords: Direct Numerical Simulation, two-phase flow, Volume of Fluid, conjugate mass transfer, two scalar approach.
\end{abstract}

\section{Introduction}

In Process Engineering, mass transfer operations based on dispersed two-phase flows are frequently applied. Typical examples are gas purification by bubbling of a gas through a liquid, oxygenation of aqueous systems in biological processes, and solvent extraction as a thermal separation process. Besides the departure from the phase equilibrium, the mass transfer depends mainly on the characteristics of the dispersed two-phase flow, i.e. on the particle size and shape, slip velocities, internal circulation, swarm behaviour etc., and on the species diffusivities. Besides experimental studies, Direct Numerical Simulations of single fluid particles, which become more and more feasible due to the ongoing increase in computational power, can be very useful since they can provide local data which usually cannot be accessed by experiments. Contrary to heat transfer, in mass transfer prob- 
lems the transported scalar - the molar concentration $c$, say- is not continuous at the interface. To handle the interfacial jump discontinuity numerically is a challenging task. Volume of Fluid (VOF)-based simulations of mass transfer across deforming interfaces have been reported in [1] and in [2, 3]. In the latter papers, transfer of oxygen from air bubbles rising in water or aqueous solutions has been simulated, taking into account the realistic jump discontinuity of the oxygen profiles at the interface. Darmana et al. [4] performed 3D simulations of mass transfer at rising fluid particles for $\mathrm{Sc}=1$ using the Front Tracking method. There, the transport resistance inside the fluid particle is neglected, i.e. a constant concentration value inside the bubble is assumed. Radl et al. [5] performed 2D simulations of deformable bubbles and bubble swarms with mass transfer in non-Newtonian liquids using a semi-Lagrangian advection scheme. To prevent stability problems, a reduced density ratio between gas and liquid is used there. Recently, first papers on numerical simulation of reactive mass transfer appeared. In $[6,7]$, the impact of single bubble wake dynamics on the reaction-enhanced mass transfer and on the yield and selectivity of the cyclohexane oxidation reaction is studied numerically for fixed shapes in 2D. In [8], reactive mass transfer at deformable interfaces is examined using a 2D Front Tracking/Front Capturing hybrid method. In [9], a Level Set based method is used to simulate mass transfer across the interface of a moving deformable droplet. This method is extended to reactive mass transfer in [10], where an instantaneous chemical reaction occurs inside a moving droplet which leads to a quasi-stationary problem for the mass transfer. In [11], 2D simulations are performed using a Front-Tracking method to investigate the effect of different Hatta and Schmidt numbers on the catalytic hydrogenation of nitroarenes for single bubbles and bubble clusters.

The focus of the present work is on a VOF-based method having the potential to be used for Direct Numerical Simulations (DNS) of mass transport in two-phase flows with deformable interfaces, including droplets, bubbles, falling films etc. For the computation of the mass transfer across the interface, two variants are employed. In the numerical study presented here, single fluid particles rising in a Newtonian fluid with mass transfer from the fluid particle to the surrounding liquid are considered.

\section{The governing equations}

In the following, we consider a fluid-particle (domain $\Omega^{d}(t)$ ) which is immersed in a liquid (domain $\Omega^{c}(t)$ ). The deformable interface between the two phases is presented as a surface of zero thickness and is denoted by $\Sigma(t)$. The transfer component $k$ has a constant initial concentration $c_{k}\left(t_{0}\right)>0$ inside the fluid particle and a zero concentration inside the surrounding liquid. Furthermore, the following assumptions are imposed:

- dilute two-phase system,

- chemically inert non surface active transfer component,

- local thermodynamical equilibrium at the interface,

- no phase change, 
- isothermal conditions,

- incompressible bulk phases.

The present paper employs a continuum mechanical model in which the governing equations are based on the conservation of mass, momentum, and (molar) mass of the transfer component. Inside the phases the transport of species $k$ is governed by the local balance equation

$$
\partial_{t} c_{k}+\nabla \cdot\left(\mathbf{u} c_{k}+\mathbf{j}_{k}\right)=R_{k} \quad \text { in } \quad \Omega^{c}(t) \cup \Omega^{d}(t) .
$$

Here $c_{k}$ is the volume specific molar concentration of the dissolved species $k$, $\mathbf{j}_{k}$ is the area specific diffusive (molecular) flux density, and $R_{k}$ is the overall reaction rate accounting for all chemical reactions in which species $k$ is involved. In the following, chemical reactions are not more considered, i.e. $R_{k}=0$. For the diffusive flux density, a suitable constitutive equation is required. Here, only diluted systems are considered. In this case the molecular transport inside the bulk phases can be described by Fick's law, i.e.

$$
\mathbf{j}_{k}=-D_{k} \nabla c_{k},
$$

with diffusion coefficient $D_{k}$. The concentration $c_{k}$ and with it the flux $\mathbf{j}_{k}$ as well as the velocity $\mathbf{u}$ are local and time dependent quantities. To solve the parabolic partial differential equations (1) inside the bulk phases, initial and suitable boundary conditions are required. The solutions inside the phases are not independent at the interface and two jump conditions are required. The first one is a transmission condition and comes from the interfacial balance. Since only non surface active transfer components are considered, the normal component of the diffusive fluxes are equal at the interface, i.e.

$$
\left[\mathbf{j}_{k}\right] \cdot \mathbf{n}_{\Sigma}=0
$$

with the jump notation

$$
[\phi]\left(\mathbf{x}_{\Sigma}\right)=\lim _{h \rightarrow+0}\left(\phi\left(\mathbf{x}_{\Sigma}+h \mathbf{n}_{\Sigma}\right)-\phi\left(\mathbf{x}_{\Sigma}-h \mathbf{n}_{\Sigma}\right)\right) .
$$

For the second interfacial condition, local thermodynamical equilibrium is assumed, i.e. the chemical potential $\mu_{k}$ of component $k$ is continuous at the interface:

$$
\left[\mu_{k}\right]=0,
$$

with

$$
\mu_{k}(T, p)=\mu_{k}^{0}\left(T, p^{0}\right)+R T \ln a_{k} .
$$

The first term in (6) is the chemical potential of component $k$ in a pure system (consisting only of component $k$ ) at temperature $T$ and standard pressure $p^{0}$, whereas the second term, containing the activity $a_{k}$ of species $k$ in a multicomponent fluid, accounts for mixing effects. For liquid systems, the activity is proportional to the 
concentration and for gas mixtures it is proportional to the partial pressure. In case of a diluted system, the thermodynamical equilibrium condition (5) reduces to

$$
c_{k \mid \Sigma}^{d}=c_{k \mid \Sigma}^{c} / m_{k}
$$

with the distribution coefficient $m_{k}=m_{k}(p, T)>0$, where $c_{k \mid \Sigma}^{d}$ and $c_{k \mid \Sigma}^{c}$ are the one-sided limits of the concentrations at the interface in the dispersed and continuous bulk phase, respectively. Since we consider only isothermal flows with small pressure gradients, the distribution coefficient $m_{k}$ is assumed to be constant. The second condition (5) is only an approximation since the deviation from the local thermodynamical equilibrium is the driving force of the mass transfer. However, this deviation is very small. Therefore, the equilibrium assumption is commonly accepted.

The underlying velocity field is governed by the two-phase Navier-Stokes equations expressing conservation of mass and momentum. Assuming continuity of the velocity at the interface, a one-field formulation is possible in which the interfacial momentum jump conditions act as source terms in the momentum equations. For viscous (Newtonian) fluids of constant density and constant surface tension, the governing equations read as

$$
\nabla \cdot \mathbf{u}=0
$$

and

$$
\rho \partial_{t} \mathbf{u}+\rho(\mathbf{u} \cdot \nabla) \mathbf{u}=-\nabla p+\eta \Delta \mathbf{u}+\rho \mathbf{g}+\sigma \kappa \mathbf{n}_{\Sigma} \delta_{\Sigma},
$$

where the momentum jump conditions are incorporated via the interfacial Delta distribution $\delta_{\Sigma}$. In this interfacial source term, $\kappa=-\nabla \cdot \mathbf{n}_{\Sigma}$ denotes the curvature (more precisely, the sum of the principal curvatures). In (9) the material properties $\rho$ and $\eta$ refer to the phase dependent values which are given as

$$
\rho=f \rho^{d}+(1-f) \rho^{c}
$$

and

$$
\eta=f \eta^{d}+(1-f) \eta^{c}
$$

where $f$ is the phase indicator function of the phase domain $\Omega^{d}(t)$.

\section{Numerical method}

For complex flow situations such as a freely moving fluid particle with a deformable interface, the mathematical model described in the previous section cannot be solved analytically but has to be treated numerically. There are several requirements for an appropriate numerical method. One challenge is the capturing of the interfacial concentration jump of the transfer component at the interface according to (7). Since a Lagrangian fluid particle cannot cross the interface (Lagrangian theorem), the species transport across the interface is purely diffusive. Therefore, the convective transport of the discontinuity shall not lead to an artificial mass transfer 
across the interface. Furthermore, the continuous-side concentration gradient normal to the interface may be very steep depending on the particle Reynolds number, $\mathrm{Re}$, and the Schmidt number, Sc, of the continuous phase. For practically relevant Sc numbers around 500, say, the concentration boundary layer cannot be resolved without specific computational techniques. The numerical scheme presented here is based on the VOF method [12] using the one-field formulation of the NavierStokes equations (9). In comparison with other free surface simulation methods, the VOF method can handle massive deformations and even topology changes as they can appear in case of large bubbles. Furthermore, the VOF method conserves the phase volume, which is an important issue if chemical reactions shall be accounted for. The phase indicator $f$ is obtained from its initial distribution by solving the advection equation

$$
\partial_{t} f+\mathbf{u} \cdot \nabla f=0
$$

In the Finite Volume context, $f$ corresponds to the volume fraction of phase $\Omega^{d}$ inside a computational cell $V$. The employed Finite Volume based VOF-solver, Free Surface 3D (FS3D) developed by Rieber [13], applies a directional as well as a kind of operating splitting. To avoid systematic errors and unsymmetries, the sequence of processed directions in the splitting scheme is altered in each time step. That is, the convective terms of all transport equations are computed firstly. Than, the forces for the momentum equations are computed and impressed before the diffusive transport terms are calculated. For the volumetric surface tension force the conservative continuum surface stress (CSS)-model of Lafaurie et al. [14] is used. The numerical solution of the discrete version of (12) is based on a geometrical based flux calculation. Application of the so called piecewise linear (or planar in case of 3D) interface calculation (PLIC) scheme for the outgoing phase volume fluxes in interfacial cells prevents interface smearing.

\subsection{Transport of molar species mass}

For the computation of the transport of a transfer species $k$, the concentration is represented by two separate scalar variables according to

$$
\phi_{k}^{d}(\mathbf{x}, t)=\left\{\begin{array}{lll}
c_{k}(\mathbf{x}, t) & \text { for } & \mathbf{x} \in \Omega^{d}(t) \\
0 & \text { for } & \mathbf{x} \in \Omega^{c}(t)
\end{array}\right.
$$

and

$$
\phi_{k}^{c}(\mathbf{x}, t)=\left\{\begin{array}{lll}
0 & \text { for } & \mathbf{x} \in \Omega^{d}(t) \\
c_{k}(\mathbf{x}, t) & \text { for } & \mathbf{x} \in \Omega^{c}(t) .
\end{array}\right.
$$

This allows for capturing the different one-sided limits of the concentrations at the interface. In the discrete Finite Volume scheme, these scalars are related to the cell 
volume $V$, i.e. in interface-containing cells the cell centered values are given as

$$
\phi_{k}^{d}(t)=\frac{1}{|V|} \int_{V \cap \Omega^{d}(t)} c_{k} d V
$$

and

$$
\phi_{k}^{c}(t)=\frac{1}{|V|} \int_{V \cap \Omega^{c}(t)} c_{k} d V
$$

\subsubsection{Convection}

The new variables are similar to the VOF-variable $f$ in that these quantities are all nonnegative. The only difference is that $\phi_{k}^{d}$ and $\phi_{k}^{c}$ can take arbitrary positive values whereas $f$ is always less or equal to one. Therefore, the convective transport of $\phi_{k}^{d}$ and $\phi_{k}^{c}$ is treated analogously to that of the convective $f$ transport, using the PLIC algorithm for the outgoing flow in interfacial cells to prevent an (artificial) convective mass transfer and a flux limiter scheme inside the bulk phases to minimize numerical diffusion.

\subsubsection{Mass transfer across the interface}

After the computation of the convective transport of species mass (and also of the other quantities like phase volume and momentum) mass transfer across the interface is calculated. Within the two scalar approach, mass transfer is accounted for by source terms. Inside a computational cell $V_{i}$ containing a part of the interface, the transferred volume specific molar mass is substracted from and added to the corresponding values of $\phi_{k, i}^{d}$ and $\phi_{k, i}^{c}$. In this notation, index $i$ stands as a short form for $(i, j, k)$ which is the full index of a grid cell in a 3D Cartesian mesh. For brevity the characters $j$ and $k$ are omitted. In the following, the calculation of onedimensional fluxes are explained only for the $x$-direction where index $i+1$ has the meaning of $(i+1, j, k)$. For the calculation of mass transfer, where the two jump conditions ( 3 ) and (7) have to be accounted for, we employ two different variants.

\section{Variant I: Equilibration of interfacial cells}

In the first variant, we assume that inside a computational cell $V_{i}$, containing a part of the interface, the transfer component $k$ is ideal mixed in the separate phases. After the convective transport both variables $\phi_{k, i}^{c}$ and $\phi_{k, i}^{d}$ have certain values which are assumed to be constant within the respective phases lying in the considered interfacial cell. However, the ratio $\phi_{k, i}^{d}\left(1-f_{i}\right) /\left(\phi_{k, i}^{c} f_{i}\right)$ does not corresponds to $m_{k}$, in general. Therefore, the characteristic of this equilibrium approach is the conservative redistribution of the (molar) species mass according to

$$
\phi_{k, i}^{d}\left|V_{i}\right|+\phi_{k, i}^{c}\left|V_{i}\right|=\phi_{k, i}^{d, e q}\left|V_{i}\right|+\phi_{k, i}^{c, e q}\left|V_{i}\right|,
$$


where the values of the variables $\phi_{k, i}^{d, e q}$ and $\phi_{k, i}^{c, e q}$ fulfil the thermodynamical condition (7). In this variant, the first transmission condition (3) is not explicitly accounted for. But this condition expresses the local (molar) mass balance at the interface which is inherently fulfilled by this approach.

Variant II: Computation of the one-sided concentration gradient

The second variant is based on the computation of the concentration gradient adjacent to the interface at the continuous side. Within an interfacial cell $V_{i}$ the total (molar) mass flux of species $k$ normal to and across the interface with interfacial area $\left|A_{\Sigma_{i}}\right|$ is given by

$$
\mathbf{j}_{\Sigma_{i}, k} \cdot \mathbf{n}_{\Sigma_{i}}\left|A_{\Sigma_{i}}\right|=\left(j_{\Sigma_{i}, k, x} n_{\Sigma_{i}, x}+j_{\Sigma_{i}, k, y} n_{\Sigma_{i}, y}+j_{\Sigma_{i}, k, z} n_{\Sigma_{i}, z}\right)\left|A_{\Sigma_{i}}\right| .
$$

Here, only directions are accounted for in which the neighbour cell is completely filled with the continuous phase. To calculate the one-dimensional diffusive flux density $j_{\Sigma_{i}, k, x}$ (where $\Sigma_{i}$ denotes the interface in cell $V_{i}, k$ the component index, and $x$ the direction) it is assumed that within the interfacial cell the dispersed phase is well mixed. Therefore, the cell centered value of $\phi_{k, i}^{d}$ is taken as the concentration value $c_{k \mid \Sigma, i}^{d}$ adjacent to the interface. Then, depending on whether cell $V_{i+1}$ or cell $V_{i-1}$ lies completely in the continuous phase, the one-dimensional flux density is computed as

$$
j_{\Sigma_{i}, k, x}=-D_{k}^{c} \frac{\phi_{k, i+1}^{c}-\phi_{k, i}^{d} /\left(f_{i} m_{k}\right)}{x_{i+1}-x_{i}}
$$

or

$$
j_{\Sigma_{i}, k, x}=-D_{k}^{c} \frac{\phi_{k, i}^{d} /\left(f_{i} m_{k}\right)-\phi_{k, i-1}^{c}}{x_{i}-x_{i-1}},
$$

respectively. The local interfacial area in an interfacial cell $V_{i}$ is calculated from the cell centered value of the gradient of the VOF-variable $f$ according to

$$
\left|A_{\Sigma, i}\right|=\|\nabla f\|_{i}\left|V_{i}\right|
$$

After calculating the cell specific total molar mass flux normal to the interface (18), the values for $\phi_{k, i}^{d}$ and $\phi_{k, i}^{c}$ are updated according to

$$
\phi_{k, i}^{d}=\phi_{k, i}^{d}-q_{k, i} \quad \text { and } \quad \phi_{k, i}^{c}=\phi_{k, i}^{c}+q_{k, i}
$$

respectively, where the source term is given by

$$
q_{k, i}=\frac{\mathbf{j}_{\Sigma_{i}, k} \cdot \mathbf{n}_{\Sigma_{i}}\left|A_{\Sigma_{i}}\right| \Delta t}{\left|V_{i}\right|}
$$


Table 1: Physical parameters

\begin{tabular}{|c|c|c|c|c|}
\hline phase & density & $\begin{array}{c}\text { dynamical } \\
\text { viscosity } \\
\eta \text { in } m P a s\end{array}$ & $\begin{array}{c}\text { diffusion } \\
\text { coefficient } \\
D \text { in } \mathrm{m}^{2} / \mathrm{s}\end{array}$ & Schmidt number \\
& $\rho$ in $\mathrm{kg} / \mathrm{m}^{3}$ & $\mathrm{~T}=\nu / \mathrm{D}$ \\
\hline dispersed & 1.2 & $18 \cdot 10^{-3}$ & $5 \cdot 10^{-6}$ & 3 \\
continuous & 1000 & 10 & $10^{-6}$ & 10 \\
\hline
\end{tabular}

\subsubsection{Diffusive transport inside the bulk phases}

Finally, diffusive transport in the bulk phases is computed. Here, especially in computational cells with a very small $f$ value it might occur that too much species mass leaves the cell during time interval $\Delta t$. The directional splitting scheme allows for a limitation of the diffusive fluxes which are calculated, using the forward differencing scheme according to

$$
\dot{N}_{k, i, i+1}=D_{k}^{c / d} \frac{c_{k, i}-c_{k, i+1}}{x_{i+1}-c_{i}}\left|A_{i, i+1}\right|,
$$

where $A_{i, i+1}$ is the cell face which connects the cells $V_{i}$ and $V_{i+1}$. This onedimensional flux is limited by the equilibrium criteria $c_{i+1}^{n+1} \geq c_{i}^{n+1}$ if $c_{i+1}^{n} \geq$ $c_{i}^{n}$ and vice versa. Furthermore, diffusive fluxes across cell faces connecting two interfacial cells are also accounted for.

\section{Simulation results and discussion}

To compare the two variants, 2D numerical simulations (i.e. with translational symmetry) of an air bubble rising in a Newtonian liquid have been performed. The area equivalent diameter of the bubble is $3 \mathrm{~mm}$, the liquid has a dynamical viscosity of 10 times higher than that of water and a density of $1000 \mathrm{~kg} / \mathrm{m}^{3}$. To keep the time step sufficiently high, a reduced surface tension of $36 \mathrm{mN} / \mathrm{m}$ has been used. For the distribution coefficient $m_{k}$ a value of 0.03 is chosen. The physical parameters are given in Table 1 . The computational domain of $1.2 \mathrm{~cm} \times 2.4 \mathrm{~cm}$ is resolved by three different computational grids; cf. Table 2. Figure 1 shows the increase of the molar mass of the transfer component within the continuous phase with time. For all resolution cases the mass transfer calculated with the equilibrium approach is higher than that calculated with the gradient approach. But the results of both variants get closer together with higher resolution. This indicates insufficient resolution of the thin concentration boundary layer; recall that the latter has a thickness proportional to $1 / \sqrt{\operatorname{Re~Sc}}$. Obviously, with the gradient approach the mass transfer is underestimated. The reason for this may be an inaccurate approximation of the interfacial area. The total interfacial area of a fluid particle is given by 
Table 2: Used numerical grids

\begin{tabular}{|c|c|c|c|}
\hline Case & number of grid cells & cell width in $\mu m$ & cells per diameter \\
\hline A & $256 \times 512$ & 46.9 & 64 \\
B & $512 \times 1024$ & 23.4 & 128 \\
C & $1024 \times 2048$ & 11.7 & 256 \\
\hline
\end{tabular}

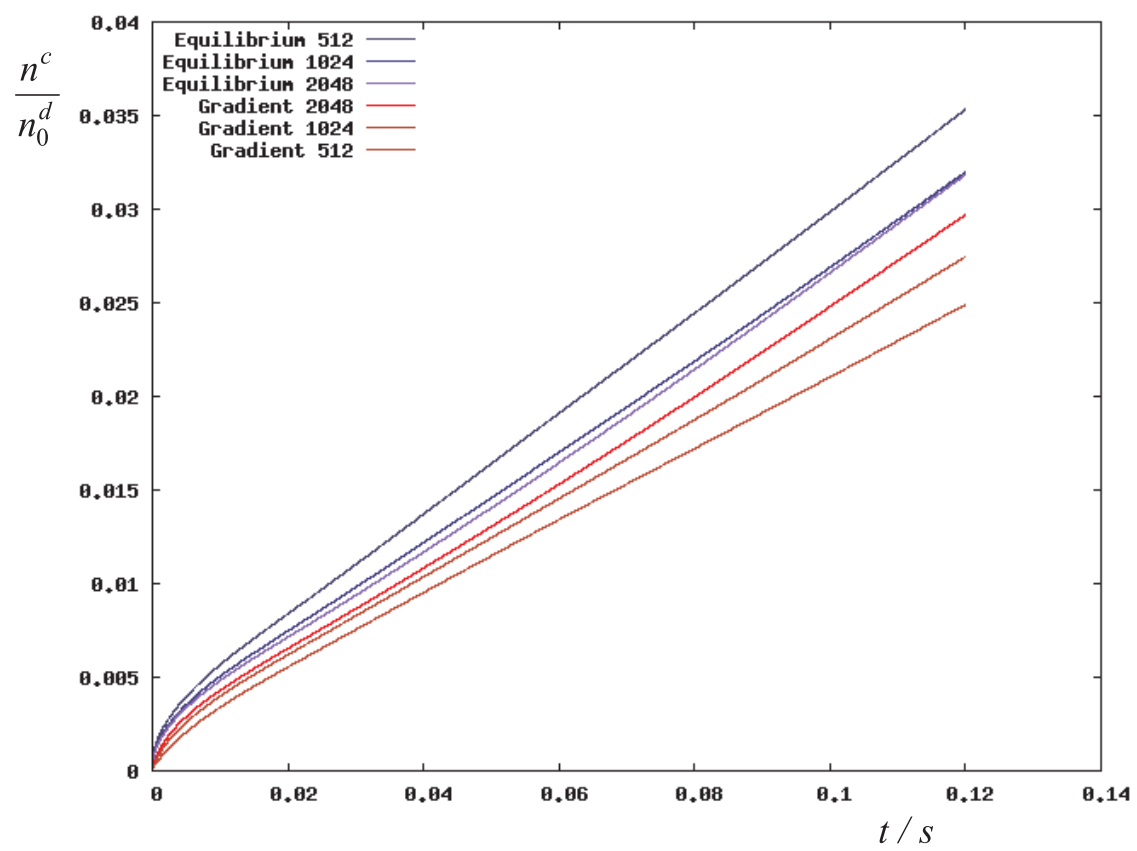

Figure 1: Molar mass of transfer species in continuous phase related to initial molar mass in fluid particle versus time

$\sum_{\text {all cells }}\|\nabla f\|_{i}\left|V_{i}\right|$. But not only interfacial cells have $\|\nabla f\|$ greater than zero but also the neighboring cells. Therefore, equation (21) yields a too low value for the local interfacial area inside a computational cell. Otherwise, the equilibrium approach inherently overestimates the mass transfer since the (local) thermodynamical equilibrium is only valid adjacent to the interface. The larger the normal distance from the interface the lower is the concentration. The assumption of ideally mixed interface cells can be interpreted as an infinitely fast molecular transport at the interface leading to a too large mass transfer. Therefore, the equilibrium approach yields an upper bound for the mass transfer. However, from Figure 1 it can be seen that grid independence is already reached. The different mass transfer 
rates are also noticeable in the concentration profiles. Figure 2 shows the concentration distribution within the continuous phase when stationary hydrodynamical conditions are reached, obtained with the two variants (left: equilibrium and right: gradient variant) at the highest resolution (case C). In both cases, species is mainly
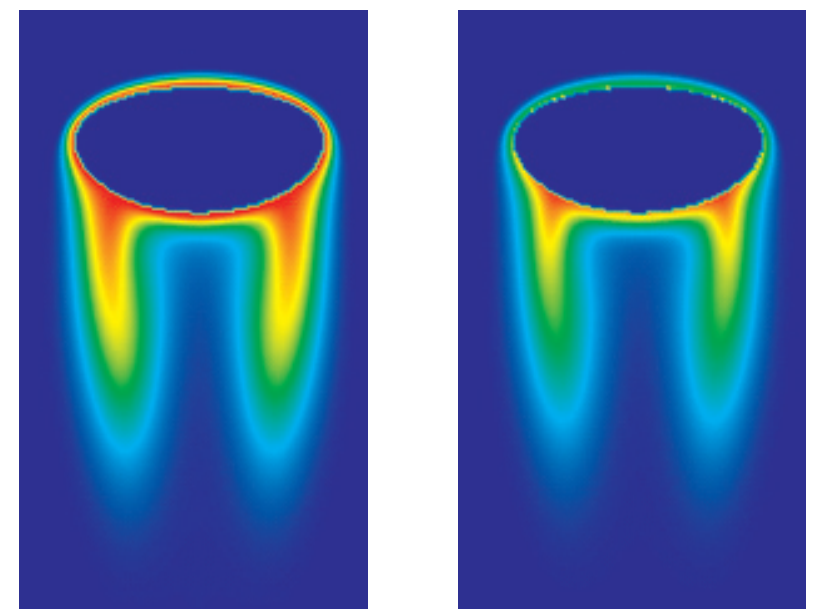

Figure 2: Concentration distribution of transfer component in the continuous phase yielded at highest resolution (Case $\mathrm{C}$ ): mass transfer calculated with equilibrium variant (left) and with the gradient variant (right)

present in the wake of the fluid particle. However, with the equilibrium approach the region of high concentrations at the stagnation points is more pronounced than those obtained with the gradient approach.

\section{Conclusions and outlook}

A new VOF-based two scalar approach for simulating the transport of chemical species within a two-phase flow is introduced. The method allows for the simulation of conjugate mass transfer problems across deformable interfaces with an arbitrary distribution coefficient. The treatment of the convective transport is analogous to that of the VOF-variable $f$, using a geometrical flux calculation. This procedure avoids artificial mass transfer due to convection. First numerical results in $2 \mathrm{D}$ at a moderate Schmidt number of 10 are performed. The results show that the mass transfer rate obtained by equilibration of the interfacial cells are always higher as those obtained by using the one-sided limit of the concentration gradient in the continuous phase. However, with finer resolution the results of both variants get closer together. For the equilibrium variant, grid independency is reached. However, the finest resolution presented here will not be sufficient for 
higher Schmidt numbers and, moreover, is not suitable for 3D simulations. The mass transfer rate obtained with the gradient variant at the highest resolution is still lower than the "true" rate. The reason for this may lie in the underestimation of the interfacial area. Therefore, further steps are the use of a more accurate interfacial area calculation scheme, the development of a subgrid model for the concentration profile at the interface, use of a moving grid technique to reduce the computational domain as well as a local grid refinement around the bubble.

\section{Acknowledgement}

We gratefully acknowledge financial support from the Deutsche Forschungsgemeinschaft (DFG) within the DFG-project "Reactive mass transfer from rising gas bubbles" (PAK-119).

\section{References}

[1] Davidson, M.R. \& Rudmann, M.J., Volume-of-fluid calculation of heat or mass transfer across deforming interfaces in two-fluid flow. Numerical Heat Transfer, B41, pp. 291-308, 2002.

[2] Bothe, D., Koebe, M., Wielage, K. \& Warnecke, H.J., VOF simulations of mass transfer from single bubbles and bubble chains rising in aqueous solutions. in Proc. 2003 ASME Joint U.S.-European Fluids Eng. Conf., 2003.

[3] Bothe, D., Koebe, M., Wielage, K., Prüss, J. \& Warnecke, H.J., Direct Numerical Simulation of Mass Transfer Between Rising Gas Bubbles and Water. Springer Verlag, Berlin, Heidelberg, New York, 2003.

[4] Darmana, D., Deen, N.G. \& Kuipers, J.A.M., Detailed 3d modelling of mass transfer processes in two-phase flows with dynamic interfaces. Chemical Engineering and Technology, 2006.

[5] Radl, S., Tryggvason, G. \& Khinast, J.G., Flow and mass transfer of fully resolved bubbles in non-newtonian fluids. AIChE Journal, 53, pp. 18611878, 2007.

[6] Khinast, J.G., Impact of 2-d bubble dynamics on the selectivity of fast gas liquid reactions. AIChE Journal, 47, pp. 2304-2319, 2001.

[7] Khinast, J.G., Koynov, A. \& Leib, T.M., Reactive mass transfer at gas-liquid interfaces: Impact of micro scale fluid dynamics on yield and selectivity of liquid phase cyclohexane oxidation. Chemical Engineering Science, 58, pp. 3961-3971, 2003.

[8] Koynov, A., Tryggvason, G. \& Khinast, J.G., Mass transfer and chemical reactions at dynamic interfaces. AIChE Journal, 51, pp. 2786-2800, 2005.

[9] Deshpande, K.B. \& Zimmermann, W.B., Simulation of interfacial mass transfer by droplet dynamics using the level-set method. Chemical Engineering Science, 61, pp. 6486-6498, 2006. 
[10] Deshpande, K.B. \& Zimmermann, W.B., Simulations of mass transfer limited reaction in a moving droplet to study transport limited characteristics. Chemical Engineering Science, 61, pp. 6424-6441, 2006.

[11] Radl, S., Koynov, A., Tryggvason, G. \& Khinast, J.G., DNS-based prediction of the selectivity of fast multiphase reactions: Hydrogenations of nitroarenes. Chemical Engineering Science, 63, pp. 3279-3291, 2008.

[12] Hirt, C.W. \& Nichols, B.D., Volume of fluid (vof) method for the dynamics of free boundaries. Journal of Computational Physics, 39, pp. 201-225, 1981.

[13] Rieber, M., Numerische Modellierung der Dynamik freier Grenzfächen in Zweiphasenströmungen. Ph.D. thesis, ITLR Universität Stuttgart, 2004.

[14] Lafaurie, B., Scardovelli, C.N., Scardovelli, R., Zaleski, S. \& G.Zanetti, Modelling merging and fragmentation in multiphase flows with surfer. Journal of Computational Physics, 113, pp. 134-147, 1994. 